\title{
Model-Dependent Constraint on Quark Total Angular Momentum Based on the Transverse Target-spin Asymmetry Measured in Deeply Virtual Compton Scattering at HERMES
}

\author{
Wolf-Dieter Nowak (on behalf of the HERMES collaboration) \\ DESY, D-15738 Zeuthen, Germany
}

\begin{abstract}
Results are reported on the transverse target-spin asymmetry (TTSA) associated with deeply virtual Compton scattering on the proton. The data have been accumulated in the years 20022004 by the HERMES experiment at DESY, in which the HERA $27.6 \mathrm{GeV}$ e+ beam scattered on a transversely polarized hydrogen target. Two azimuthal amplitudes of the TTSA appearing to LO in $1 / \mathrm{Q}$ and $\alpha_{s}, A_{U T}^{\sin \left(\phi-\phi_{S}\right) \cos \phi}$ and $A_{U T}^{\cos \left(\phi-\phi_{S}\right) \sin \phi}$, are given as a function of $-t, x_{B}, Q^{2}$ in the kinematic range $|t|<0.7 \mathrm{GeV}^{2}, 0.03<x_{B}<0.35$ and $1<Q^{2}<10 \mathrm{GeV}^{2}$. The first amplitude is found to be sensitive to the generalized parton distribution (GPD) $E$ of the proton, which can be parameterized in a GPD model through quark total angular momentum $J_{q}(q=u, d)$. Within the context of this model, a constraint in the $\left(J_{u}, J_{d}\right)$ plane is obtained from HERMES TTSA data.
\end{abstract}

Keywords: proton structure, GPDs, DVCS, hard exclusive, transverse target-spin asymmetry PACS: $13.60 . \mathrm{Fz}, 13.60 . \mathrm{Hb}, 13.60 . \mathrm{Le}, 13.88 .+\mathrm{e}, 14.20 . \mathrm{Dh}$

\section{DEEPLY VIRTUAL COMPTON SCATTERING}

Hard exclusive leptoproduction of a real photon off a nucleon, by the virtual photon emitted by the incoming lepton, is called deeply virtual Compton scattering (DVCS). This process is the presently most practical tool to constrain generalized parton distributions (GPDs) which provide a detailed description of the structure of the nucleon. Great interest in GPDs arose after it was realized that the total angular momentum carried by quarks in the nucleon, $J_{q}$, may be accessed once the GPDs $H_{q}$ and $E_{q}$ for the quark species $q$ have been constrained by experimental data [1].

The DVCS final state is experimentally indistinguishable from that of the BetheHeitler $(\mathrm{BH})$ process, in which the real photon is radiated from the incoming or scattered lepton. Hence their amplitudes add coherently and the cross section contains an interference term $I$ :

$$
\frac{d \sigma}{d x_{B} d Q^{2} d|t| d \phi} \propto\left|\tau_{B H}\right|^{2}+\left|\tau_{D V C S}\right|^{2}+\underbrace{\tau_{D V C S} \tau_{B H}^{*}+\tau_{D V C S}^{*} \tau_{B H}}_{I} .
$$

Here $x_{B}$ represents the Bjorken scaling variable, $-Q^{2}$ the virtual-photon fourmomentum squared, and $-t$ the squared four-momentum transfer to the target. The azimuthal angle $\phi$ is measured between the scattering plane, spanned by the incoming and outgoing leptons, and the production plane made up by the virtual and real photons. 
While at HERMES energies the BH cross section dominates over the DVCS cross section, the DVCS amplitude can still be investigated by studying the interference term $I$. This task is accomplished by measuring various azimuthal cross section asymmetries.

\section{TRANSVERSE TARGET-SPIN ASYMMETRY}

A transverse single target-spin asymmetry (TTSA) associated with DVCS on the proton can be measured with an unpolarized lepton beam (U) and a transversely polarized (T) hydrogen target [2]:

$$
A_{U T}\left(\phi, \phi_{S}\right)=\frac{d \sigma\left(\phi, \phi_{S}\right)-d \sigma\left(\phi, \phi_{S}+\pi\right)}{d \sigma\left(\phi, \phi_{S}\right)+d \sigma\left(\phi, \phi_{S}+\pi\right)}
$$

Here $\phi_{S}$ is the azimuthal orientation of the target polarization vector with respect to the scattering plane. At leading order in $\alpha_{S}$ and $1 / Q$, the 2 azimuthal TTSA amplitudes are:

$$
\begin{aligned}
A_{U T}^{\sin \left(\phi-\phi_{S}\right) \cos \phi} & \propto \operatorname{Im}\left[F_{2} H-F_{1} E\right], \\
A_{U T}^{\cos \left(\phi-\phi_{S}\right) \sin \phi} & \propto \operatorname{Im}\left[F_{2} \widetilde{H}-F_{1} \xi \widetilde{E}\right] .
\end{aligned}
$$

The Compton form factors $H, E, \widetilde{H}$ and $\widetilde{E}$ are convolutions of the respective GPDs $H$, $E, \widetilde{H}$ and $\widetilde{E}$ with hard scattering kernels, while $F_{1}$ and $F_{2}$ are the Dirac and Pauli form factors of the proton, respectively. The skewness parameter $\xi$ is given by $\xi \simeq \frac{x_{B}}{2-x_{B}}$.

Direct access to total quark angular momentum $J_{q}(q=u, d)$ is made possible with a GPD model [3] that parameterizes the GPD $E$ using $J_{u}$ and $J_{d}$ as free parameters. Within

the context of this GPD model the TTSA amplitude $A_{U T}^{\sin \left(\phi-\phi_{S}\right) \cos \phi}$ is found to be sensitive to $J_{u}$ and $J_{d}$ and insensitive to the other model parameters [2].

In this paper first results are reported, obtained at the HERMES experiment, on the TTSA associated with DVCS on the proton. Furthermore, a model-dependent constraint on $J_{u}$ vs $J_{d}$ is extracted by comparing the TTSA results of HERMES with theoretical predictions based on the above mentioned GPD model.

\section{DEEPLY VIRTUAL COMPTON SCATTERING AT HERMES}

HERMES is a fixed-target experiment using the $27.6 \mathrm{GeV}$ longitudinally polarized electron or positron beam of the HERA accelerator at DESY Hamburg. The HERMES forward spectrometer [4] is instrumented with tracking chambers providing momentum and angular measurements for charged particles. Lepton-hadron separation is achieved by a transition-radiation detector, a pre-shower counter and an electromagnetic calorimeter. The latter detects both the scattered lepton and the photon produced in the BH/DVCS process. As recoil proton detection was not possible for the analyzed data sets, kinematic requirements are imposed on scattered lepton and produced real photon in order to ensure the exclusivity of the reaction. In particular, the requirement $-(1.5)^{2}<M_{x}^{2}<(1.7)^{2}$ $\mathrm{GeV}^{2}$ is imposed on the missing mass, as determined from Monte-Carlo simulations which include the finite resolution of the spectrometer, by comparing signal and background distributions. 


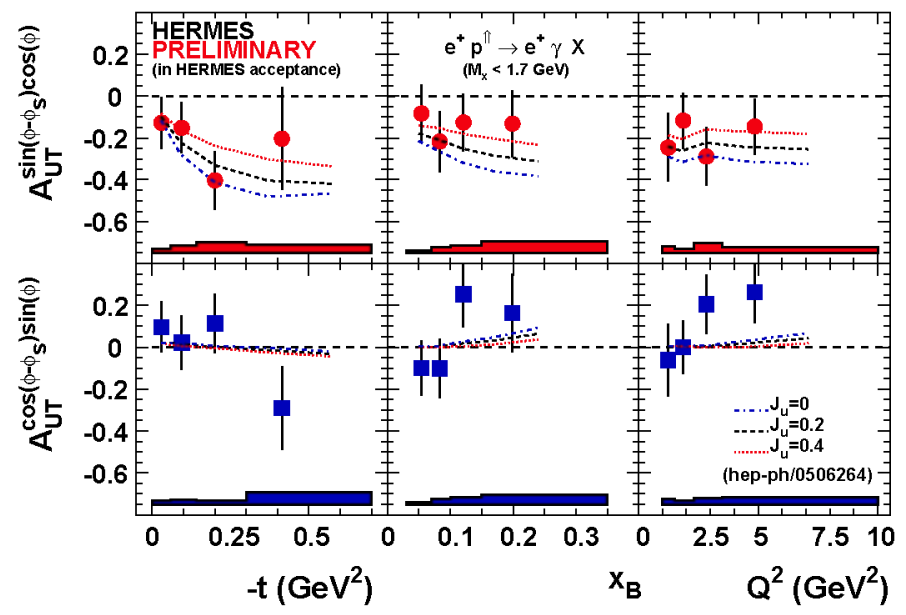

FIGURE 1. The $A_{U T}^{\sin \left(\phi-\phi_{S}\right) \cos \phi}$ and $A_{U T}^{\cos \left(\phi-\phi_{S}\right) \sin \phi}$ amplitudes of the transverse target-spin asymmetry associated with DVCS, shown as a function of $-t, x_{B}$ and $Q^{2}$ for the exclusive sample $\left(-(1.5)^{2}<M_{x}^{2}<\right.$ $(1.7)^{2} \mathrm{GeV}^{2}$ ) after background correction. The error bars (bands) represent the statistical (systematic) uncertainties. The curves are the predictions from a GPD model with different $u$-quark total angular momentum $J_{u}$ and fixed $d$-quark total angular momentum $J_{d}=0$ [2].

\section{RESULTS}

Figure 1 shows the two TTSA amplitudes $A_{U T}^{\sin \left(\phi-\phi_{S}\right) \cos \phi}$ and $A_{U T}^{\cos \left(\phi-\phi_{S}\right) \sin \phi}$ as a function of $-t, x_{B}$ and $Q^{2}$, as extracted from HERMES data accumulated in 2002-2004 using a transversely polarized hydrogen target. Corrections for background and smearing have been applied. The main contributions to the systematic uncertainty are those from the determination of the target polarization and in the background correction. Also shown in figure 1 are theoretical predictions based on a phenomenological model of GPDs [3]. For both amplitudes, the experimental results are in general agreement with the theoretical predictions.

Sensitivity to $J_{u}$ is seen only for the first amplitude. Hence the integrated TTSA result $\left\langle A_{U T}^{\sin \left(\phi-\phi_{S}\right) \cos \phi}\right\rangle=-0.149 \pm 0.058$ (stat.) \pm 0.033 (syst.), extracted from the 2002-2004 data at the average kinematics $\langle-t\rangle=0.12 \mathrm{GeV}^{2},\left\langle x_{B}\right\rangle=0.095,\left\langle Q^{2}\right\rangle=2.5 \mathrm{GeV}^{2}$, is input to a fit in order to constrain $J_{u}$ and $J_{d}$. The reduced $\chi^{2}$ value, defined as

$$
\Delta \chi^{2}=\chi^{2}-\chi_{\text {minimum }}^{2}=\left[A^{\exp }-A^{V G G}\left(J_{u}, J_{d}\right)\right]^{2} /\left[\delta A_{\text {stat }}^{2}+\delta A_{\text {syst }}^{2}\right] .
$$

is calculated on a grid for $J_{u}$ and $J_{d}$. Here $A^{\exp }$ denotes the measured (integrated) TTSA amplitude, $\delta A_{\text {stat }}\left(\delta A_{\text {syst }}\right)$ its statistical (systematic) uncertainty, and $A^{V G G}$ is the value calculated at the average kinematics by a code [5] based on the above mentioned GPD model [3].

The one-standard-deviation constraint on $J_{u}$ vs $J_{d}$ is defined as the area in the ( $J_{u}$, $\left.J_{d}\right)$-plane, in which the reduced $\chi^{2}$ value is below one: $J_{d} / 2.9+J_{u}=0.42 \pm 0.21 \pm 0.06$ 


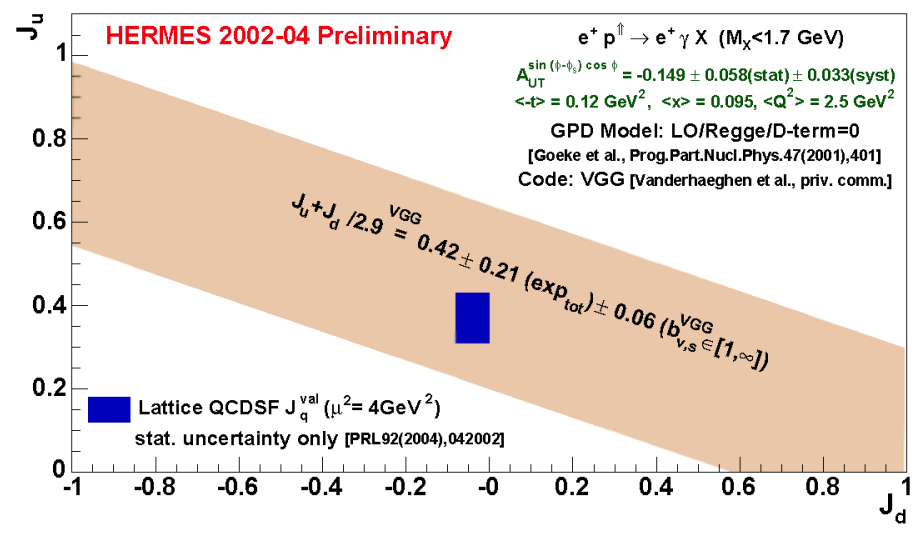

FIGURE 2. Model-dependent constraint on $u$-quark total angular momentum $J_{u}$ vs $d$-quark total angular momentum $J_{d}$, obtained by comparing the experimental result and theoretical predictions on the TTSA amplitude $A_{U T}^{\sin \left(\phi-\phi_{S}\right) \cos \phi}$. Also shown is a Lattice result from the QCDSF collaboration, obtained at the scale $\mu^{2}=4 \mathrm{GeV}^{2}$ for valence quark contributions only.

(see Fig. 2). The first uncertainty is due to the experimental uncertainty in the measured TTSA amplitude. The second one is a model uncertainty, obtained by varying from one to infinity the unknown profile parameter $b$ which controls the skewness dependence of GPDs [3]. The $t$-dependence of GPDs is modelled using the Regge ansatz [3]. The impact of using it or its alternative - the factorized ansatz - on the theoretical predictions on the TTSA amplitudes has been found to be negligible [2]. The D-term contribution to the GPDs $H$ and $E$ is set to zero, as suggested by the HERMES results on the beamcharge asymmetry [6]. If the D-term were modelled according to the chiral quark soliton model [3], the resulting constraint is shifted to $J_{d} / 2.9+J_{u}=0.53 \pm 0.21 \pm 0.06$.

\section{ACKNOWLEDGMENTS}

This work has been supported by the German Bundesministerium für Bildung und Forschung (BMBF) (contract nr. 06 ER 125I) and the European Community-Research Infrastructure Activity under the FP6 "Structuring the European Research Area" program (HadronPhysics I3, contract nr. RII3-CT-2004-506078).

\section{REFERENCES}

1. X. Ji, Phys. Rev. Lett. 78 (1997) 610, Phys. Rev. D55 (1997) 7114.

2. F. Ellinghaus et al., arXiv:hep-ex/0506264, Eur. Phys. J. C46 (2006) 729.

3. K. Goeke et al., Prog. Part. Nucl. Phys. 47 (2001) 401.

4. HERMES Coll., K. Ackerstaff et al., Nucl. Instr. and Meth. A417 (1998) 230.

5. M. Vanderhaeghen, P.A.M. Guichon and M. Guidal, priv. comm., 2003.

6. HERMES coll., A. Airapetian et al., submitted to Phys. Rev. D, hep-ex/0605108. 